\title{
Lymphome non hodgkinien primaire à cellules B larges de la cavité orale : à propos d'un cas clinique
}

\section{Non-Hodgkin lymphoma B-cell primary of the oral cavity: about a clinical case}

\section{Résumé}

\section{MOTS-CLEFS :}

- Lymphome, chimiothérapie, cavité orale

\section{KEYWORDS:}

- Lymphoma, chemotherapy, oral corvity

$\operatorname{AOS} n^{\circ} 285-2017$
Introduction : Les lymphomes sont des tumeurs malignes des lignées cellulaires de lymphocytes. Ils sont principalement classés en : lymphome de Hodgkin (LH), et non hodgkinien (LNH). La différence entre les deux types est anatomopathologique. Le LH est caractérisé par la présence de cellules de Reed-Sternberg, alors que l'absence de ces cellules et la présence d'autres cellules lymphoïdes néoplasiques est nécessaire pour le diagnostic du LNH.

Discussion : Il s'agit d'un homme âgé de 45 ans, sans antécédents particuliers, consultant pour une tuméfaction palatine indolore, évoluant depuis deux mois avec asymétrie faciale. Le patient rapportait aussi une obstruction nasale droite. L'examen clinique endobuccal a révélé la présence d'une tuméfaction maxillaire souple et indolore ò la palpation, et recouverte d'une muqueuse érythémateuse. Cette tuméfaction est située au niveau du secteur molaire droit, s'étendant à la région palatine.

$\grave{A}$ l'examen radiologique panoramique nous avons noté la présence d'une lyse osseuse intéressant le secteur maxillaire droit. Par ailleurs le cône beam a montré une effraction bucco-sinusienne droite associée ò un comblement du sinus droit.

Une biopsie a été réalisée au niveau du site concluant à un lymphome non hodgkinien à cellule B larges.

Conclusion : Le lymphome non hodgkinien de la cavité orale est une lésion rare, son étiologie n'est pas encore élucidée. Ses aspects cliniques et radiologiques ne sont pas spécifiques. La confirmation du diagnostic est anatomopathologique.

\section{Abstract}

Intruduction: Lymphomas are malignant tumors of lymphocyte cell lines. They are mainly classified as : Hodgkin's lymphoma (LH), and not Hodgkin's lymphoma (NHL). The difference between the two types is anatomopathological. $L H$ is characterized by the presence of Reed-Sternberg cells, whereas the absence of these cells and the presence of other neoplastic lymphoid cells is necessary for the diagnosis of NHL.

Discussion: This is a 45-year-old man with no previous history, consulting for painless palatal swelling, evolving for two months with facial asymmetry. The patient also reported a right nasal obstruction. The endobuccal clinical examination revealed the presence of soft and painless maxillary tumefaction on palpation and covered with an erythematous mucosa. This swelling is located at the level of the right molar sector and extending to the palatal region. In the panoramic radiological examination we noticed the presence of a bone lysis involving the right maxillary area. Moreover the cone beam showed a straight bucco-sinusian intrusion associated with a filling of the right sinus. A boipsy was performed at the site finding a non-Hodgkin lymphoma with large $B$ cells.

Conclusion: Non-Hodgkin's lymphoma of the oral cavity is a rare lesion, its etiology is not yet elucidated. Its clinical and radiological aspects are not specific. Confirmation of diagnosis is anatomopathological. 


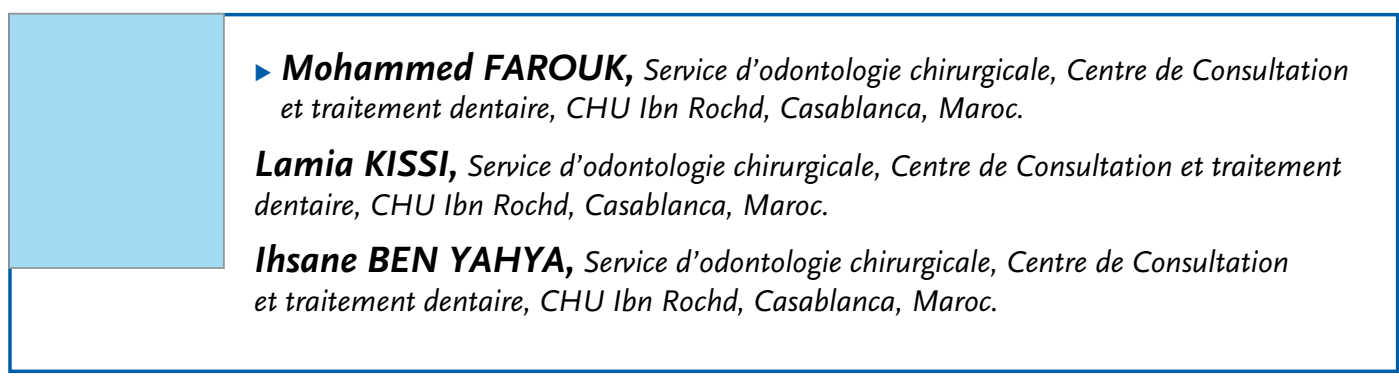

\section{INTRODUCTION}

Les lymphomes sont des tumeurs malignes des lignées cellulaires de lymphocytes. Ils sont principalement classés en : lymphome de Hodgkin, et non hodgkinien $[1,4]$. Le lymphome non hodgkinien (LNH) provient de l'évolution maligne et de la prolifération de cellules lymphoïdes de type $\mathrm{B}$ ou $\mathrm{T}$. Il se produit généralement dans les ganglions lymphatiques, mais 20 à $30 \%$ des sites peuvent êtres extra-nodaux [5, 10].

La cavité orale constitue un rare site extranodal primaire du lymphome malin non Hodgkinien. Les sujets de sexe masculin sont les plus touchés par cette tumeur $[3,4]$.

\section{CAS CLINIQUE}

Il s'agit d'un homme âgé de 45 ans, sans antécédents particuliers, consultant pour une tuméfaction palatine indolore, évoluant depuis deux mois avec asymétrie faciale. Le patient rapportait aussi une obstruction nasale droite.

L'examen clinique exobuccal a noté la présence d'une tuméfaction jugale droite, érythémateuse et douloureuse à la palpation (Fig. 1).

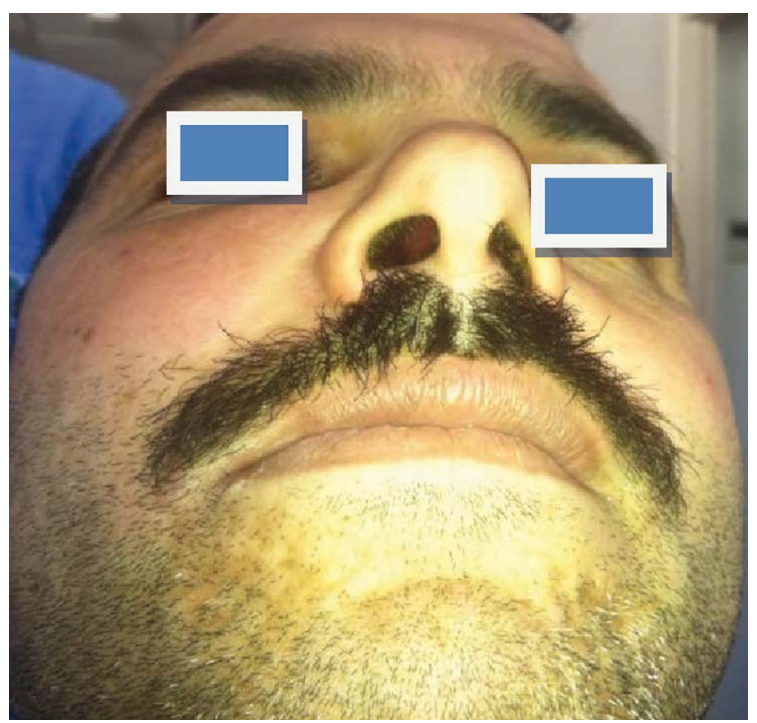

$\triangle$ Fig. 1 : Examen exobuccal : asymétrie faciale.
L'examen clinique endobuccal a révélé la présence d'une tuméfaction maxillaire souple et indolore à la palpation, et recouverte d'une muqueuse érythémateuse. Cette tuméfaction est située au niveau du secteur molaire droit et s'étendant à la région palatine (Fig. 2).

À l'examen radiologique panoramique nous avons noté la présence d'une lyse osseuse intéressant le secteur maxillaire droit. Par ailleurs le cône beam a montré une effraction bucco-sinusienne droite associée à un comblement du sinus droit (Fig. 3a, 3b).

À ce stade, le diagnostic d'une lésion maligne était fortement évoqué, vu les aspects cliniques et radiologiques de cette lésion.

Une biopsie à été réalisée au niveau du site.

L'examen histologique et immunohistochimique a confirmé le diagnostic du lymphome non hodgkinien. Les cellules lymphomateuses ont une grande taille et un noyau avec une chromatine claire contenant un ou plusieurs nucléoles; de nombreuses mitoses sont visibles (Fig. 4).

Le patient à été adressé au service d'hématologie, la prise en charge a consisté en une chimiothérapie.

Une rémission a été notée après 6 mois, le contrôle du site de la lésion a montré une régression de la tuméfaction et de l'asymétrie faciale (Fig. 5).

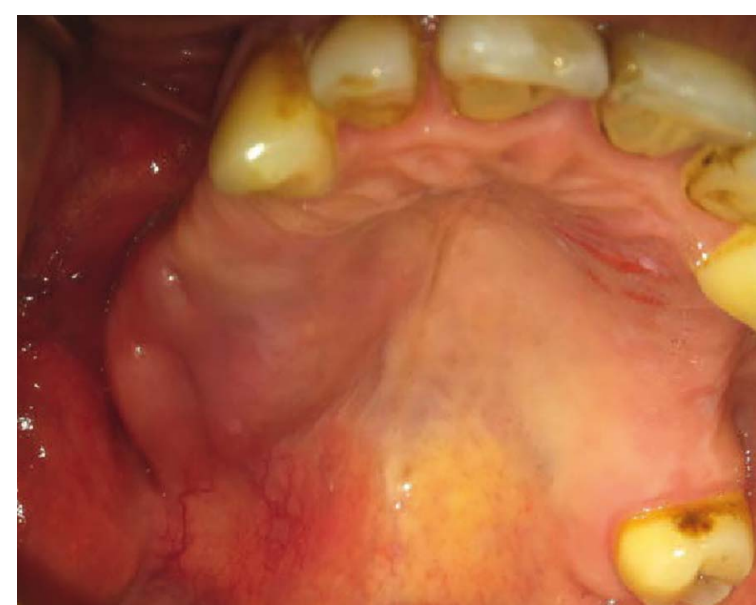

Fig. 2: Examen endobuccal: tuméfaction palatine droite souple et indolore à la palpation, et recouverte d'une muqueuse érythémateuse. 

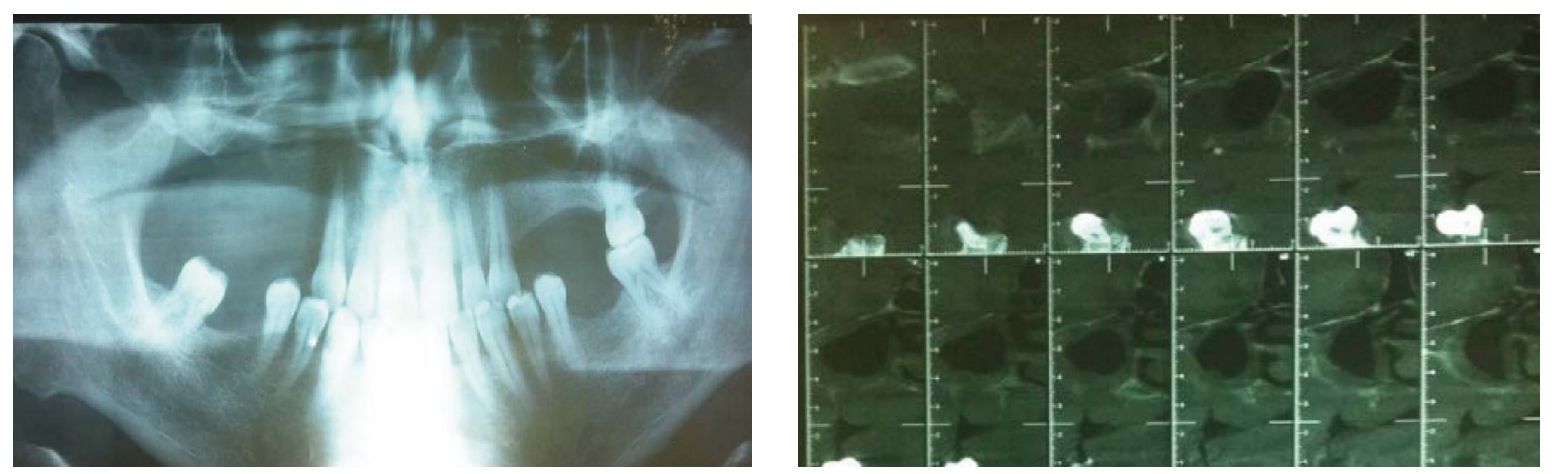

$\triangle$ Fig. 3 : Examen radiologique, 3a. panoramique, 3b. cône beam : lyse osseuse au niveau du secteur maxillaire droit associée à une effraction et un comblement sinusien droit.

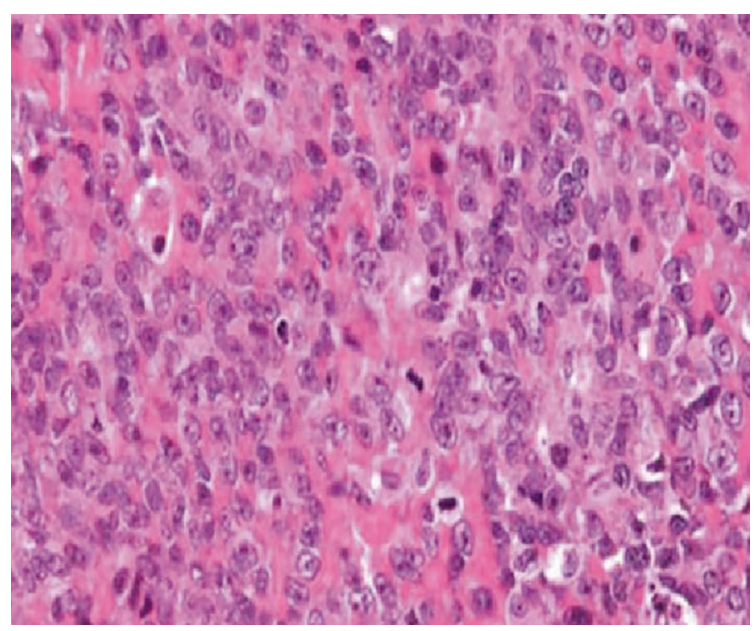

$\triangle$ Fig. 4 : Examen anatomopathologique : cellules lymphomateuses Type B de grande taille, avec un noyau avec une chromatine claire contenant un ou plusieurs nucléoles; de nombreuses mitoses sont visibles.

\section{DISCUSSION}

Les lymphomes sont un groupe de néoplasmes issus $\mathrm{du}$ système lymphoréticulaire principalement les ganglions lymphatiques, caractérisé par la prolifération anormale des cellules lymphoïdes $\mathrm{B}$ et $\mathrm{T}$ ou de leurs précurseurs. C'est une tumeur maligne qui présente, après le carcinome épidermoide, le deuxième cancer de la région de la tête et du cou, avec un pourcentage de $2,6 \%$ [1].

Les lymphomes sont classés en deux groupes : les lymphomes Hodgkiniens (LH) et non hodgkiniens (LNH). La différence entre les deux types est anatomopathologique. Le LH est caractérisé par la présence de cellules de Reed-Sternberg, alors que l'absence de ces cellules et la présence d'autres cellules lymphoïdes néoplasiques est nécessaire pour le diagnostic du LNH [2].

Généralement, les lymphomes se développent aux niveaux des ganglions, mais peuvent impliquer des sites extra-nodaux comme, le thymus, la rate, le foie, le tractus gastro-intestinal et la cavité orale [1].

Les patients de sexe masculin âgés de plus de 40 ans sont les plus atteints par cette maladie $[3,4]$.

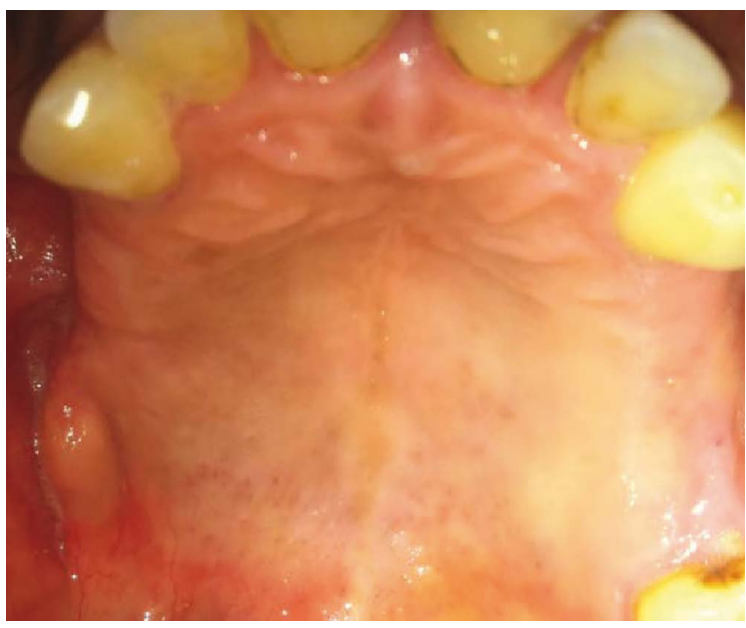

$\triangle$ Fig. 5 : Contrôle après rémission montrant une régression de la lésion.

Pour le groupe du LNH, jusqu'à $40 \%$ sont observés dans un site extra-ganglionnaire. La localisation orale est extrêmement rare, et ne représente que 2-3\% de ces cas extra ganglionnaires $[2,5,6]$.

Dans sa forme orale, le LNH peut siéger au niveau du tissu osseux ou des parties molles [7, 8].

Les sites les plus communs au niveau de la cavité orale sont le palais mou, les amygdales et les glandes salivaires accessoires. D'autres localisations sont très rares notamment la gencive et le périapex. $[4,7,9,10]$. Pour notre cas, la localisation était palatine.

Il existe plusieurs sous types histologiques du LNH. Certaines études ont montré pour la forme orale - une prédominance du sous-type à cellules B large, qui représente $50 \%$ à $68 \%$ de l'ensemble LNH oraux [2]. C'est le cas de notre patient.

Le LNH est caractérisé par sa croissance localisée, cependant le retard du diagnostic peut favoriser sa diffusion $[3,4,11]$.

L'étiologie du LNH n'est pas encore claire [11], plusieurs causes ont été rapporté dans la littérature : L'irradiation, une immunosuppression à long terme, $y$ compris les maladies auto-immunes et la polyarthrite 
rhumatoïde, le lupus érythémateux systémique, le syndrome de Sjögren, et certaines infections, y compris le virus d'Epstein-Barr (EBV), le virus lymphotrope des cellules T humaines 1 (HTLV-1), le VIH, Helicobacter pylori, Chlamydia, et herpesvirus- 8 humain (HHV-8, KSHV) [12, 13].

Les patients infectés par le VIH sont plus à risque de développer un LNH. Le lymphome à cellules B agressif est l'une des variantes les plus couramment observées chez ces individus [12].

Cliniquement, les lésions buccales peuvent apparaître comme une zone érythémateuse, une tuméfaction indolore, souvent associée à une ulcération de surface secondaire à un traumatisme, ou à une mobilité dentaire $[7,12]$. Si le site est mandibulaire une paresthésie peut être observée [10].

L'aspect radiologique n'est pas spécifique, la lésion est souvent associée à une lyse osseuse dont l'étendue dépend du stade du diagnostic $[3,4]$. La localisation maxillaire peut être associée à une invasion sinusale. La localisation mandibulaire quand à elle, peut être associée à un élargissement du canal alvéolaire inférieur ou du foramen mentonnier si la maladie intéresse ces sites [10].

L'absence de spécificités clinique et radiologique de cette maladie est la cause de confusion avec des lésions bénignes notamment, la maladie parodontale, les péricoronarites, l'abcès parodontal [10].

La confirmation du diagnostic est anatomopathologique. Elle est basée sur deux critères : l'absence des cellules de Reed-Sternberg, et la présence de cellules lymphoïdes néoplasiques plus ou moins différenciées. Ce sont des cellules lymphoïdes géantes avec de grands noyaux vésiculaires, des nucléoles proéminents et un cytoplasme basophile abondant. L'anatomopathologie permet aussi de déterminer le sous type de la maladie selon la nature de ces cellules $[3,4,11,13,14]$.

Le traitement dépend de plusieurs facteurs : le soustype histologique, le siège, et la taille de la tumeur [3, $8,1]$.

Le traitement est basé sur la chimiothérapie, la radiothérapie et la chirurgie dans diverses combinaisons [8]. Le traitement standard comprend une combinaison de chimiothérapie avec le cyclophosphamide, le methotrexate, la doxorubicine, la vincristine, la cytarabine arabinoside, et le prednisone [8].

La radiothérapie adjuvante normalement livrée à un niveau de 40-50 Gy [10], est particulièrement utile dans des variantes histopathologiques suggérant un comportement agressif [7].

La chirurgie associée à la radiothérapie est indiquée pour les formes localisées non agressives [15].

Le pronostic dépend de plusieurs facteurs notamment le stade du diagnostic, le type de la maladie et l'état du patient [11]. Cependant une récidive est possible même plusieurs années après la rémission $[8,11]$.

\section{CONCLUSION}

Le lymphome non hodgkinien de la cavité orale est une lésion rare, son étiologie n'est pas encore élucidée. Ses aspects cliniques et radiologiques ne sont pas spécifiques. La confirmation du diagnostic est anatomopathologique.

Le rôle du médecin dentiste est important dans le diagnostic de cette lésion qui doit être évoqué devant toute tuméfaction indolore, à évolution rapide, associée à une résorption osseuse étendue avec effractions des zones anatomiques avoisinantes. 


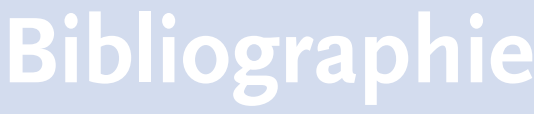

[1] Batra R, Kaur H, Jindal S. Extranodal large B-cell type aggressive non-Hodgkin's lymphoma. Indian J Dent. 2014 Oct ; 5(4) : 225-8.

[2] Souto GR, Pereira TS, Castro AF, Mesquita RA. Diffuse large B-cell lymphoma, not otherwise specified of the palate : A case report. JClin Exp Dent. 2013 Dec 1 ; 5(5) : e287-90.

[3] P. Koblera J, Borcicb I, Filipovic Zorea M, Nolac D. SerticdPrimary non-Hodgkin's lymphoma of the oral cavity. Oral Oncology Extra. Volume 41, Issue 1, January 2005, Pages 12-14.

[4] Guevara-Canales JO, Morales-Vadillo R, Cava-Vergiú CE, Leite FP, Miranda Chaves Netto HD, Soares FA, Chaves Md. Survival in patients with oral and maxillofacial diffuse large B-cell lymphoma. Braz Oral Res. 2013 JulAug ; 27 (4) : 349-55.

[5] London J, Grados A, Fermé C, Charmillon A, Maurier F, Deau B, Crickx E, Brice P, Chapelon-Abric C, Haioun C, Burroni B, Alifano M, Le Jeunne C, Guillevin L, Costedoat-Chalumeau N, Schleinitz N, Mouthon L, Terrier B. Sarcoidosis occurring after lymphoma : report of 14 patients and review of the literature. Medicine (Baltimore). 2014 Nov; 93 (21) : e121.

[6] Bolukbas F, Kutluturkan S. Symptoms and symptom clusters in non Hodgkin's lymphoma patients in Turkey. Asian Pac J Cancer Prev. 2014 ; 15 (17).

[7] Parihar S, Garg RK, Narain P. Primary extra-nodal non-Hodgkin's lymphoma of gingiva : A diagnostic dilemma. J Oral Maxillofac Pathol. 2013 May ; 17 (2) : 320.

[8] Vinoth PN, Selvan SM, Sahni L, Krishnaratnam K, Rajendiran S, Anand CV, Scott JX. Primary extra nodal non-Hodgkin's lymphoma of the oral cavity in a young girl. Natl J Maxillofac Surg. 2012 Jul ; 3 (2) : 187-9.

[9] Jaradat JM, Potluri A, Bilodeau EA. B-cell lymphoma, unclassifiable, with features intermediate between diffuse large B-cell lymphoma and Burkitt lymphoma : report of a case in the oral cavity. Indian J Dent Res. 2013 May-Jun ; 24 (3) : 384-6.

[10] Wong GB, Spadafora S, Barbon N, Caputo M. Primary extranodal B-cell non-Hodgkin lymphoma mimicking an endodontic lesion : report of 2 cases. J Can Dent Assoc. $2013 ; 79$ : d93.
[11] Pía López-Jorneta, Ambrosio BermejoFenollb. Oral mucosal non-Hodgkin's lymphoma. Oral Oncology Extra. Volume 41, Issue 6, July 2005, Pages 101-103.

[12] Augustine D, Sekar B, Thiruneervannan R, Sundhar M, Reddy DV, Patil SG. Primary oral non-Hodgkin's lymphoma - A clinicopathologic study with immunohistochemical analysis. J Int Soc Prev Community Dent. 2014 Nov ; 4 (Suppl 1) : S68-71.

[13] Deyhimi P, Keshani F, Azmoudeh F, Hashemzadeh Z. Metastatic anaplastic large cell lymphoma of the oral cavity. Dent Res J (Isfahan). 2012 Dec ; 9 (Suppl 1) : S127-31.

[14] Patil AV, Deshpande RB, Kandalgaonkar SM, Gabhane MH1. Diffuse large B-cell lymphoma (extranodal) of maxillary buccal vestibule. J Oral Maxillofac Pathol. 2015 May-Aug;19(2) : 270.

[15] Ashok V Patil, Rashmi B Deshpande, Shilpa M Kandalgaonkar, and Mahesh $\mathbf{H}$ Gabhane. Diffuse large B-cell lymphoma (extranodal) of maxillary buccal vestibule. J Oral Maxillofac Pathol. 2015 May-Aug; 19(2) : 270 . 